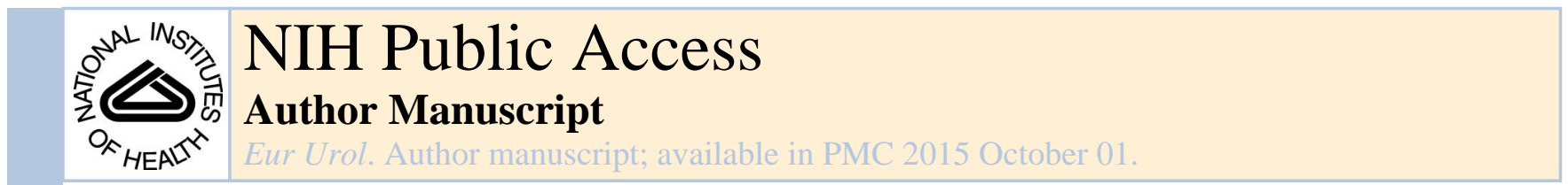

Published in final edited form as:

Eur Urol. 2014 October ; 66(4): 646-652. doi:10.1016/j.eururo.2014.01.018.

\title{
The Influence of Prior Abiraterone Treatment on the Clinical Activity of Docetaxel in Men with Metastatic Castration-resistant Prostate Cancer
}

\author{
Michael T. Schweizer ${ }^{a}$, Xian C. Zhou $^{\mathrm{a}}$, Hao Wang ${ }^{\mathrm{a}}$, Sunakshi Bassi ${ }^{\mathrm{b}}$, Michael A. Carducci ${ }^{\mathrm{a}}$, \\ Mario A. Eisenberger ${ }^{a}$, and Emmanuel S. Antonarakis ${ }^{a},{ }^{*}$ \\ aThe Sidney Kimmel Comprehensive Cancer Center, Johns Hopkins University, Baltimore, MD, \\ USA \\ bJohns Hopkins University, Baltimore, MD, USA
}

\section{Abstract}

Background-Taxanes may partly mediate their effect in castration-resistant prostate cancer (CRPC) through disruption of androgen-receptor trafficking along microtubules. This raises the possibility of cross-resistance between androgen-directed agents and docetaxel.

Objective-To evaluate docetaxel efficacy after abiraterone treatment in CRPC patients.

Design, setting, and participants-This was a single-institution, retrospective analysis in CRPC patients $(N=119)$ who either received abiraterone before docetaxel (AD) $(n=24)$ or did not receive abiraterone before docetaxel (docetaxel only; $n=95$ ). Men initiated docetaxel between December 2007 (the date abiraterone was first used at our center) and May 2013.

(C) 2014 European Association of Urology. Published by Elsevier B.V. All rights reserved.

*Corresponding author. Prostate Cancer Research Program, Sidney Kimmel Comprehensive Cancer Center, Johns Hopkins University School of Medicine, 1650 Orleans Street, CRB1-1M45, Baltimore, MD, 21231, USA. Tel.: +1 410502 7528, Fax: +1 4106148397. eantona1@jhmi.edu (E. Antonarakis).

Author contributions: Emmanuel S. Antonarakis had full access to all the data in the study and takes responsibility for the integrity of the data and the accuracy of the data analysis.

Study concept and design: Schweizer, Antonarakis.

Acquisition of data: Schweizer, Bassi.

Analysis and interpretation of data: Schweizer, Zhou, Wang, Antonarakis.

Drafting of the manuscript: Schweizer, Antonarakis.

Critical revision of the manuscript for important intellectual content: Wang, Carducci, Eisenberger.

Statistical analysis: Zhou, Wang.

Obtaining funding: Antonarakis, Wang.

Administrative, technical, or material support: Antonarakis, Wang.

Supervision: Antonarakis.

Other (specify): None.

Financial disclosures: Emmanuel S. Antonarakis certifies that all conflicts of interest, including specific financial interests and relationships and affiliations relevant to the subject matter or materials discussed in the manuscript (eg, employment/ affiliation, grants or funding, consultancies, honoraria, stock ownership or options, expert testimony, royalties, or patents filed, received, or pending), are the following: M. A. Carducci has been a paid consultant for Sanofi. E. S. Antonarakis, X. C. Zhou, and H. Wang are partially funded by the US National Institutes of Health (grant P30 CA006973).

Publisher's Disclaimer: This is a PDF file of an unedited manuscript that has been accepted for publication. As a service to our customers we are providing this early version of the manuscript. The manuscript will undergo copyediting, typesetting, and review of the resulting proof before it is published in its final citable form. Please note that during the production process errors may be discovered which could affect the content, and all legal disclaimers that apply to the journal pertain. 
Outcome measurements and statistical analysis-The primary efficacy end points were prostate-specific antigen (PSA) progression-free survival (PFS) and clinical/radiographic PFS on docetaxel. Differences between groups were assessed using univariate and multivariable analyses.

Results and limitations-Men in the AD group had a significantly higher risk for progression than those in the docetaxel-only group. Median PSA PFS was 4.1 mo in the AD group and 6.7 mo in the docetaxel-only group $(p=0.002)$. Median PFS was also shorter in the AD group (4.4 mo vs $7.6 \mathrm{mo} ; p=0.003)$. In multivariable analysis, prior abiraterone treatment remained an independent predictor of shorter PSA PFS (hazard ratio [HR]: 3.48; 95\% confidence interval [CI], 1.36-8.94; $p$ $=0.01$ ) and PFS (HR: $3.62 ; 95 \%$ CI, 1.41-9.27; $p=0.008$ ). PSA declines $250 \%$ were less frequent in the $\mathrm{AD}$ group ( $38 \%$ vs $63 \% ; p=0.02$ ). The small size and retrospective nature of this study may have introduced bias.

Conclusions-Men receiving abiraterone before docetaxel were more likely to progress on docetaxel and less likely to achieve a PSA response than abiraterone-naïve patients. Crossresistance between abiraterone and docetaxel may explain these findings; however, larger, more definitive studies are still needed to confirm this.

\section{Keywords}

Abiraterone; Activity; Docetaxel; Efficacy; Progression-free survival; Prostate cancer

\section{Introduction}

Prostate cancer (PCa) remains a major health concern, resulting in nearly 260000 annual deaths worldwide [1]. Since Charles Huggins first described the effects of surgical castration on men with metastatic PCa in the 1940s, PCa has been recognized as an androgenresponsive disease [2,3]. To date, androgen ablation, either through surgical or medical castration (ie, gonadotropin-releasing hormone $[\mathrm{GnRH}]$ agonist/antagonist therapy), has remained the cornerstone of advanced PCa management. Unfortunately, nearly all men progress in spite of castrate testosterone levels, at which point they are described as having castration-resistant prostate cancer (CRPC) (Fig. 1) [4]. In 2004, docetaxel became the first agent shown to produce survival gains in the CRPC setting [5,6]. More recently, a number of additional, second-line, androgen-directed therapies (ie, abiraterone and enzalutamide) have been shown to prolong life in men with metastatic CRPC [7-10]. At this juncture, the question of how to best sequence these agents with one another and the issue of crossresistance between them remain inadequately addressed. The realization that a large proportion of men who develop CRPC remain dependent on androgen/androgen-receptor (AR) signaling has led to a renewed interest in the AR as a therapeutic target. As a result, a number of newer AR-directed therapies have been developed. Mechanistically, these agents primarily work through ligand depletion (eg, abiraterone) or through interference with AR trafficking and signaling (eg, enzalutamide) [7]. When combined with a GnRH agonist, abiraterone decreases testosterone from the castrate level $(<50 \mathrm{ng} / \mathrm{dl})$ to the undetectable level ( $<1 \mathrm{ng} / \mathrm{dl})$ through CYP-17 enzyme inhibition. Abiraterone was initially approved in the postdocetaxel window; however, more recently it has also been approved for prechemotherapy use [8,9]. To this end, in the last 1-2 yr, abiraterone use has increased in the prechemotherapy space. However, because the original phase 3 docetaxel data were 
published in the preabiraterone era, it has been questioned whether the efficacy of docetaxel might be negatively affected by prior abiraterone treatment $[5,6]$.

Furthermore, laboratory studies have shown that taxanes may be at least partly effective in the treatment of PCa by disrupting microtubule-mediated AR nuclear trafficking [11-15]. In addition to in vitro and in vivo work supporting this purported mechanism of action, there are also microarray and circulating tumor-cell clinical data that support docetaxel's ability to inhibit AR nuclear translocation. In humans, docetaxel has been shown to significantly decrease nuclear localization of the AR compared to docetaxel-naïve controls. Furthermore, through analyzing the circulating tumor cells of subjects treated with docetaxel, Darshan and colleagues demonstrated that patients with PSA progression (>25\% PSA increase) on docetaxel were significantly more likely to have nuclear AR localization compared to those with stable or docetaxel-responsive ( $230 \%$ PSA decline) disease [14]. These observations raise the possibility of cross-resistance between taxanes and androgen-directed agents such as abiraterone and enzalutamide.

In the case of ketoconazole, a less potent CYP-17 inhibitor than abiraterone, its effects on subsequent responses to docetaxel have been mixed. In subjects pretreated with ketoconazole, one group of investigators reported trends toward shortened progression-free survival (PFS) and overall survival (OS) on docetaxel, while another group found no difference in PFS or OS based on prior ketoconazole treatment status [16,17]. Finally, a small $(n=35)$, single-arm, retrospective analysis of patients treated with abiraterone followed by docetaxel found lower-than-expected docetaxel activity when compared to historic controls [18]. Therefore, the impact of prior abiraterone therapy on subsequent responsiveness to docetaxel remains poorly defined. We hypothesized that in men with metastatic CRPC, the administration of abiraterone prior to docetaxel would lead to impaired docetaxel efficacy compared with patients that were abiraterone-naïve at the time of docetaxel initiation. Herein, we report the results of a retrospective analysis in patients from a single, large academic center who either had or had not received abiraterone prior to docetaxel treatment. By using data from the abiraterone era, we aim to provide a contemporary perspective on the interaction between these two important agents.

\section{Patients and methods}

\subsection{Patients}

This was a retrospective, single-institution analysis that included all metastatic CRPC patients treated with standard docetaxel chemotherapy who either received abiraterone prior to docetaxel treatment (AD) or did not (docetaxel only). CRPC was defined on the basis of evidence of disease progression (clinical, radiographic, or PSA elevation) in spite of castrate serum testosterone levels and continuous GnRH agonist/antagonist therapy. Only patients treated with docetaxel between December 2007 (the first date of abiraterone use at our center) and May 2013 were included in this analysis to ensure a contemporaneous control group. Follow-up data were collected through October 2013.

Subjects treated with docetaxel who carried a diagnosis of metastatic PCa were identified through our oncology pharmacy. Additional clinical data were derived through chart review 
of our electronic medical records. Subjects treated with docetaxel for anything other than metastatic CRPC (eg, as adjuvant therapy or for small cell prostatic carcinoma) or those in whom we were unable to reliably confirm the chemotherapy course (eg, dates of therapy or abiraterone pretreatment status were not documented) were excluded. Institutional review board approval was obtained prior to data collection.

\subsection{Statistical analysis}

The primary objective was to determine the effect of prior abiraterone treatment on time to PSA progression and clinical/radiographic progression after docetaxel initiation. PSA PFS was defined as the time interval from docetaxel initiation to first PSA progression. PSA progression was defined as a rising PSA level while on docetaxel that was $225 \%$ and $\geq 2$ $\mathrm{ng} / \mathrm{ml}$ above the baseline or nadir value (Prostate Cancer Working Group 2 [PCWG2] definition) [4]. In most instances, this was confirmed at a subsequent date; however, confirmation was not consistently performed on all patients. PFS was defined as the time interval from docetaxel initiation until radiographic or clinical progression (PCWG2 definition) or death, whichever came first [4]. Confirmatory scans were not generally performed since patients were treated per regular clinical practice. PSA elevations alone were not considered in the definition of PFS. Subjects were censored upon initiating a new therapy subsequent to docetaxel if they did not display evidence of clinical/radiographic progression by that time.

Descriptive statistics were used to summarize the patient characteristics of the two groups, and differences between cohorts were compared using the $t$ test for continuous variables or chi-square test or Barnard exact test for categorical variables. PSA PFS and PFS were summarized using the Kaplan-Meier method, and differences between groups were determined via a log-rank test. Univariate Cox proportional hazards models and multivariable models were constructed to evaluate the effect of prior abiraterone treatment on PSA PFS and PFS. Covariates included in the multivariable model included treatment group (ie, AD vs docetaxel only), use of an antiosteoclast agent, Eastern Cooperative Oncology Group (ECOG) performance status, pain, the presence of liver/lung metastases, baseline PSA level, and the number of bone metastases. All tests were two-sided and considered significant at $p \leq 0.05$. Statistical analyses were conducted using $\mathrm{R}$ software v. 2.15.1 (R Project for Statistical Computing, Bethesda, MD, USA), and Stata v.10.0 (StataCorp LP, College Station, TX, USA).

\section{Results}

\subsection{Patients}

Between December 2007 and May 2013, 140 men with PCa received docetaxel-based chemotherapy. Twenty-one were excluded secondary to not meeting the eligibility requirements of the study. Reasons for exclusion included docetaxel given for non-CRPC (eg, as adjuvant therapy) $(n=9)$, docetaxel given as therapy for a concurrent malignancy or for small cell prostatic carcinoma $(n=7)$, and treatment course incompletely documented (eg, unknown docetaxel initiation date) $(n=5)$. This resulted in a final cohort of 119 men who received standard docetaxel chemotherapy for metastatic CRPC. Twenty-four of these 
patients (20\%) received abiraterone before docetaxel (AD group), and 95 (80\%) were abiraterone-naïve (docetaxel-only group). After a median follow-up of $5.5 \mathrm{mo}$ (95\% confidence interval [CI], 4.8-6.4 mo) after receiving docetaxel, 20 subjects (83\%) in the AD group and $76(80 \%)$ in the docetaxel-only group developed PSA progression. Nineteen subjects (79\%) in the AD group and 70 (74\%) in the docetaxel-only group progressed by radiographic or clinical criteria (excluding PSA elevations). Notable differences between these two groups included ECOG performance status, the presence of lung metastases, number of bone metastases, and baseline PSA level (Table 1). Differences in the proportion of men receiving an antiandrogen (eg, bicalutamide) or ketoconazole before docetaxel were nonsignificant between groups.

\subsection{Docetaxel activity after abiraterone}

Patients in the AD group were at significantly higher risk for both PSA and clinical/ radiographic progression on docetaxel. In patients pretreated with abiraterone, univariate analysis revealed a hazard ratio (HR) of 2.21 for PSA PFS (95\% CI, 1.13-3.52; $p=0.002$ ), and an HR of 2.22 for PFS (95\% CI, 1.31-3.78; $p=0.003$ ) while on docetaxel. PSA PFS and PFS were significantly shorter in patients pretreated with abiraterone (Fig. 2). The median PSA PFS for the AD and docetaxel-only groups were $4.1 \mathrm{mo}$ (95\% CI, 2.8-5.8 mo) and $6.7 \mathrm{mo}(95 \% \mathrm{CI}, 5.3-7.6 \mathrm{mo})$, respectively $(p=0.002)$. The median PFS for the AD group was $4.4 \mathrm{mo}(95 \% \mathrm{CI}, 3.1-6.7 \mathrm{mo}$ ) versus $7.6 \mathrm{mo}$ (95\% CI, 6.2-8.4 mo) for the docetaxel-only group $(p=0.003)$.

Baseline differences between the AD and docetaxel-only groups were controlled for by constructing a multivariable model. This multivariable analysis revealed that the association of prior abiraterone treatment with increased risk for progression on docetaxel persisted with respect to both PSA PFS and PFS. For those in the AD arm, multivariable analysis revealed an HR of 3.48 for PSA PFS (95\% CI, 1.36-8.94; $p=0.01$ ) and an HR of 3.62 for PFS (95\% CI, 1.41-9.27; $p=0.008)$. No other clinical characteristics included in our multivariable model (ie, use of an antiosteoclast agent, pain score, performance status, baseline PSA level, presence of visceral metastases, or number of bone metastases) were found to significantly associate with PSA PFS or PFS (Table 2).

PSA responses ( $250 \%$ declines in PSA level) to docetaxel were also less frequent in the AD group (38\% vs 63\%; $p=0.02$ ). Waterfall plots depicting changes in PSA level after docetaxel in the docetaxel-only and AD groups are presented in Figure 3. In addition, of the 23 subjects in the AD group with available PSA response data to both abiraterone and docetaxel, $11(48 \%)$ failed to demonstrate a PSA response to both abiraterone and docetaxel. However, seven subjects (30\%) who did not demonstrate a PSA response to abiraterone did subsequently achieve a PSA response to docetaxel.

\section{Discussion}

In this exploratory study, we analyzed progression data after docetaxel treatment in men with metastatic CRPC who either did or did not receive prior abiraterone treatment. While our analysis is somewhat limited by its small sample size, our findings do support our initial hypothesis that abiraterone pretreatment leads to decreased responsiveness to docetaxel. Of 
note, a prior, single-arm, retrospective report by Mezynski and colleagues reached the same conclusion [18]. This report builds on that study by directly comparing outcomes after docetaxel treatment between contemporary cohorts of abiraterone-naïve and abirateronepretreated patients. It is notable that in our analysis, as well as in the one by Mezynski et al, that the observed median time to PSA progression (ie, PSA PFS) was quite similar at 4.1 mo and 4.6 mo, respectively; this speaks to the validity of our PSA PFS estimate in abirateronepretreated patients. Importantly, we observed PSA responses to docetaxel in almost onethird of subjects who previously had no PSA response to abiraterone, suggesting that a proportion of abiraterone-unresponsive patients may still derive a benefit from docetaxel.

While ideally this type of analysis would evaluate which sequence of administering abiraterone (ie, before or after docetaxel) leads to the longest OS, this was not feasible due to the small number of death events and the relatively short follow-up. In addition, during the period that this study was conducted, a number of new agents (eg, sipuleucel-T, enzalutamide, cabazitaxel) were approved for use after docetaxel treatment for men with CRPC on the basis of demonstrable survival gains $[10,19,20]$. Controlling for these agents' effect on OS independent of the sequence with which abiraterone was given was not feasible. It should be noted that we did assess for differences in the predocetaxel treatment with ketoconazole or antiandrogens (agents commonly used before docetaxel in this population) and found no significant difference between the two groups, indicating that therapies received prechemotherapy were unlikely to affect our results. Additionally, given the imbalances in baseline characteristics between the two groups, it remains possible that differences in disease severity may have influenced the time to progression. One would expect, however, that if time to progression were only a function of baseline characteristics, and not influenced by cross-resistance between abiraterone and docetaxel, that the effect of docetaxel on PSA characteristics would be similar between groups. The fact that PSA PFS was significantly different between the docetaxel-only and AD groups supports our initial hypothesis. When considered in the context of the aforementioned work showing that docetaxel may, at least partly, exert its antitumor effect in CRPC through inhibition of AR trafficking via microtubule inhibition, these clinical data support the argument for crossresistance between taxanes and AR-targeting agents [11-15]. Furthermore, these differences in PSA progression and clinical/radiographic progression on docetaxel persisted after differences between groups were accounted for through multivariable analysis.

This analysis has several limitations. First, our abiraterone-pretreated cohort was small, with only 24 subjects (and 20 PSA progression events). This may have led to a low precision in estimating our HRs, as evidenced by wide CIs (Table 2). Second, despite our efforts, there is still the possibility that our analysis suffered from lead-time bias, given that the AD group was almost certainly further along in their disease course upon inclusion in this study. Third, given that this was a retrospective analysis, allocation between cohorts was nonrandom. While the control group was contemporary to the time period that abiraterone was used at our institution, a recent trend toward using abiraterone before docetaxel has been seen at our center and elsewhere. This trend is most appreciated by the fact that the earliest date of docetaxel initiation in the AD group was November 2011, while the latest date of docetaxel initiation in the docetaxel-only group was September 2012. Given that there is $<1 \mathrm{yr}$ of overlap between the date of docetaxel initiation between these groups speaks to a shift in 
practice patterns occurring after abiraterone's US Food and Drug Administration approval in April 2011. Finally, given that the definition for radiographic progression was not prospectively defined, differences in treatment practices among the various physicians caring for those men included in this study may have affected the accuracy of our PFS estimates. In addition to nonuniform scanning intervals, confirmatory CT scans and/or bone scans were not generally ordered, and this the true PCWG2 criteria for defining progression were not strictly adhered to [4,21]. Nonetheless, these data reflect the real-world experience in managing men with metastatic CRPC on docetaxel chemotherapy.

\section{Conclusions}

In spite of its limitations, this study represents the only comparative analysis of PSA PFS and PFS after docetaxel treatment for patients who either have or have not received prior abiraterone. These findings support prior work raising the concern for cross-resistance between the CYP-17 inhibitors and docetaxel, and provide valuable information regarding which patients are likely to derive the most benefit from docetaxel. These findings require confirmation in a larger cohort of patients; however, if confirmed, it stands to reason that docetaxel might be more helpful as an upfront therapy in a defined subset of CRPC patients (eg, those requiring rapid pain palliation, with bulky visceral disease, or with rapidly expanding disease).

In conclusion, this report represents the strongest available evidence to date that a clinically meaningful cross-resistance between abiraterone and docetaxel exists. This finding needs to be confirmed by other investigators and should be expanded to other novel AR-directed therapies, such as enzalutamide.

\section{Acknowledgments}

Funding/Support and role of the sponsor: None.

\section{References}

1. Jemal A, Bray F, Center MM, Ferlay J, Ward E, Forman D. Global cancer statistics. CA Cancer J Clin. 2011; 61:69-90. [PubMed: 21296855]

2. Huggins C, Hodges CV. Studies on prostatic cancer: I. The effect of castration, of estrogen and of androgen injection on serum phosphatases in metastatic carcinoma of the prostate. 1941. J Urol. 2002; 168:9-12. [PubMed: 12050481]

3. Huggins C, Hodges CV. Studies on prostatic cancer. I. The effect of castration, of estrogen and of androgen injection on serum phosphatases in metastatic carcinoma of the prostate 1941. J Urol. 2002; 167:948-51. [PubMed: 11905923]

4. Scher HI, Halabi S, Tannock I, et al. Design and end points of clinical trials for patients with progressive prostate cancer and castrate levels of testosterone: recommendations of the Prostate Cancer Clinical Trials Working Group. J Clin Oncol. 2008; 26:1148-59. [PubMed: 18309951]

5. Tannock IF, de Wit R, Berry WR, et al. Docetaxel plus prednisone or mitoxantrone plus prednisone for advanced prostate cancer. N Engl J Med. 2004; 351:1502-12. [PubMed: 15470213]

6. Petrylak DP, Tangen CM, Hussain MH, et al. Docetaxel and estramustine compared with mitoxantrone and prednisone for advanced refractory prostate cancer. N Engl J Med. 2004; 351:1513-20. [PubMed: 15470214] 
7. Schweizer MT, Antonarakis ES. Abiraterone and other novel androgen-directed strategies for the treatment of prostate cancer: a new era of hormonal therapies is born. Ther Adv Urol. 2012; 4:16778. [PubMed: 22852027]

8. De Bono JS, Logothetis CJ, Molina A, et al. Abiraterone and increased survival in metastatic prostate cancer. N Engl J Med. 2011; 364:1995-2005. [PubMed: 21612468]

9. Ryan CJ, Smith MR, de Bono JS, et al. Abiraterone in metastatic prostate cancer without previous chemotherapy. N Engl J Med. 2013; 368:138-48. [PubMed: 23228172]

10. Scher HI, Fizazi K, Saad F, et al. Increased survival with enzalutamide in prostate cancer after chemotherapy. N Engl J Med. 2012; 367:1187-97. [PubMed: 22894553]

11. Zhu ML, Horbinski CM, Garzotto M, Qian DZ, Beer TM, Kyprianou N. Tubulin-targeting chemotherapy impairs androgen receptor activity in prostate cancer. Cancer Res. 2010; 70:79928002. [PubMed: 20807808]

12. Cheetham P, Petrylak DP. Tubulin-targeted agents including docetaxel and cabazitaxel. Cancer J. 2013; 19:59-65. [PubMed: 23337758]

13. Gan L, Chen S, Wang Y, et al. Inhibition of the androgen receptor as a novel mechanism of taxol chemotherapy in prostate cancer. Cancer Res. 2009; 69:8386-94. [PubMed: 19826044]

14. Darshan MS, Loftus MS, Thadani-Mulero M, et al. Taxane-induced blockade to nuclear accumulation of the androgen receptor predicts clinical responses in metastatic prostate cancer. Cancer Res. 2011; 71:6019-29. [PubMed: 21799031]

15. Thadani-Mulero M, Nanus DM, Giannakakou P. Androgen receptor on the move: boarding the microtubule expressway to the nucleus. Cancer Res. 2012; 72:4611-5. [PubMed: 22987486]

16. Pond GR, Armstrong AJ, Galsky MD, Wood BA, Leopold L, Sonpavde G. Efficacy of docetaxelbased chemotherapy following ketoconazole in metastatic castration-resistant prostate cancer: Implications for prior therapy in clinical trials. Urol Oncol. 2013; 31:1457-63. [PubMed: 22552048]

17. Aggarwal R, Halabi S, Kelly WK, et al. The effect of prior androgen synthesis inhibition on outcomes of subsequent therapy with docetaxel in patients with metastatic castrate-resistant prostate cancer: results from a retrospective analysis of a randomized phase 3 clinical trial (CALGB 90401). Cancer. 2013; 119:3636-43. [PubMed: 23913744]

18. Mezynski J, Pezaro C, Bianchini D, et al. Antitumour activity of docetaxel following treatment with the CYP17A1 inhibitor abiraterone: clinical evidence for cross-resistance? Ann Oncol. 2012; 23:2943-7. [PubMed: 22771826]

19. Kantoff PW, Higano CS, Shore ND, et al. Sipuleucel-T immunotherapy for castration-resistant prostate cancer. N Engl J Med. 2010; 363:411-22. [PubMed: 20818862]

20. De Bono JS, Oudard S, Ozguroglu M, et al. Prednisone plus cabazitaxel or mitoxantrone for metastatic castration-resistant prostate cancer progressing after docetaxel treatment: a randomised open-label trial. Lancet. 2010; 376:1147-54. [PubMed: 20888992]

21. Eisenhauer EA, Therasse P, Bogaerts J, et al. New response evaluation criteria in solid tumours: revised RECIST guideline (version 1. 1). Eur J Cancer. 2009; 45:228-47. [PubMed: 19097774] 


\section{Patient summary}

We examined the efficacy of docetaxel in castration-resistant prostate cancer patients who either did or did not receive prior abiraterone. We found that men receiving abiraterone before docetaxel were less likely to achieve a PSA response and were more likely to progress sooner on docetaxel than abiraterone-untreated patients. This may be due to cross-resistance. 


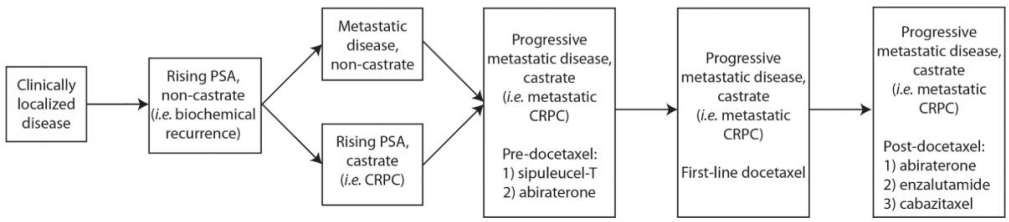

Death due to competing causes

Death due to prostate cancer

Fig. 1.

Prostate cancer clinical states model (adapted from Scher et al [4]). 


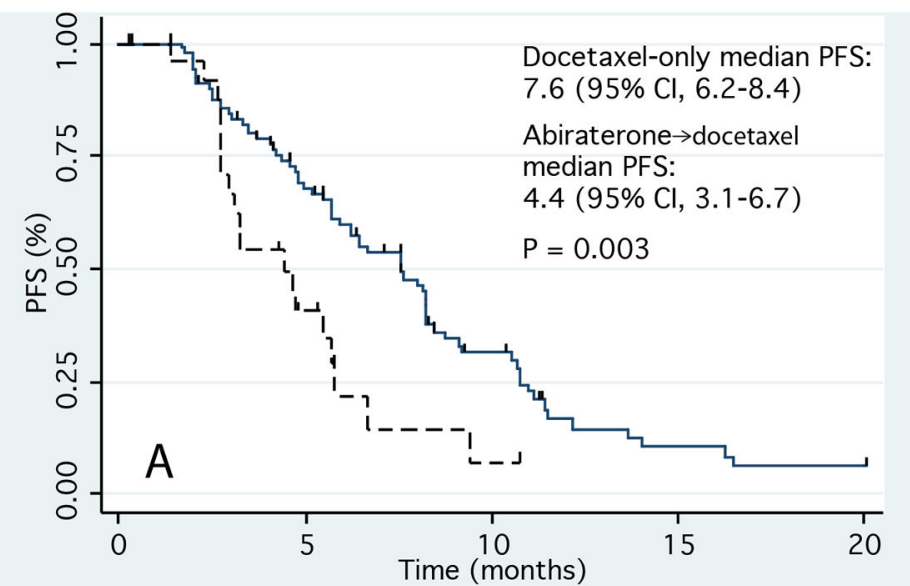

Number at risk

\begin{tabular}{l} 
Docetaxel-only \\
Abiraterone $\rightarrow$ docetaxel \\
\cline { 2 - 2 }
\end{tabular}

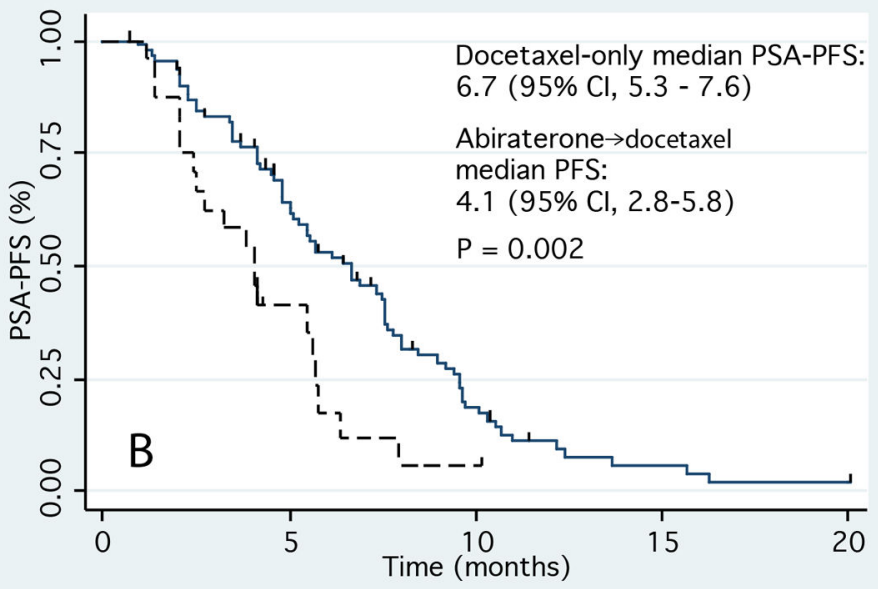

Number at risk

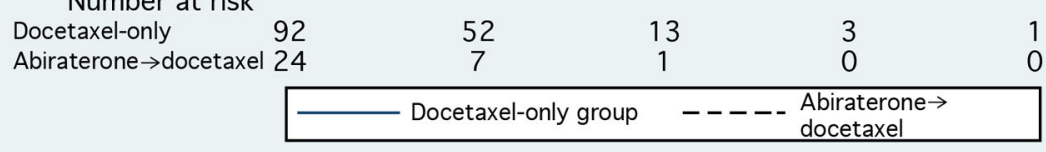

Fig. 2.

Kaplan-Meier curves for (a) progression-free survival (PFS) and (b) prostate-specific antigen PFS during treatment with docetaxel.

Abiraterone $\rightarrow$ docetaxel $=$ abiraterone-pretreated group; $\mathrm{CI}=$ confidence interval; docetaxelonly = abiraterone-naïve group; PSA = prostate-specific antigen. 


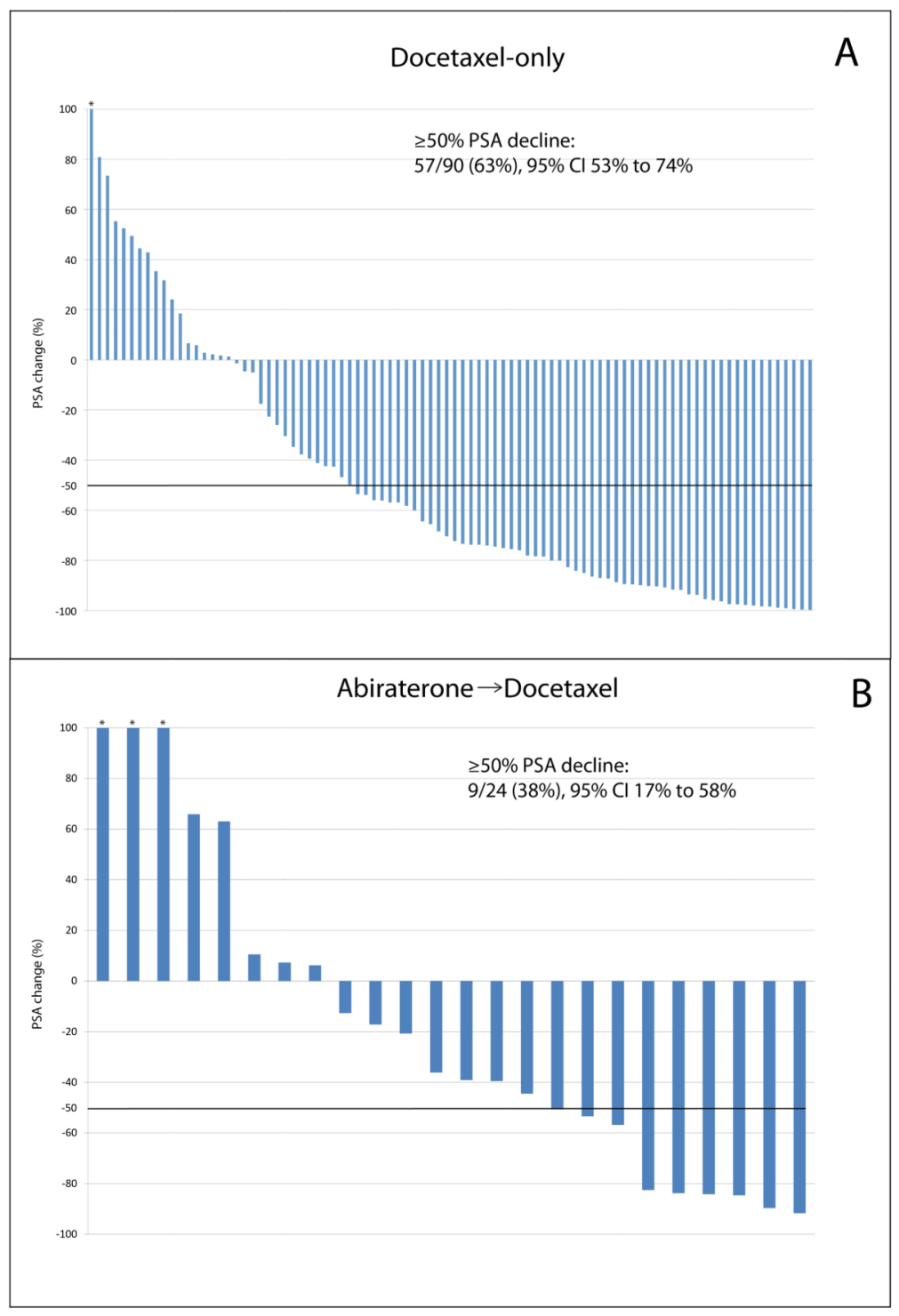

Fig. 3.

Waterfall plots depicting the maximal prostate-specific antigen (PSA) decline (or minimum increase for those without a PSA decline) after docetaxel treatment for the (a) abirateronenaïve (docetaxel only) and (b) abiraterone-pretreated cohort.

* Bar is truncated due to $>100 \%$ PSA increase.

$\mathrm{CI}=$ confidence interval. 
Table 1

Baseline demographic characteristics at the time of docetaxel initiation

\begin{tabular}{|c|c|c|c|}
\hline & Abiraterone pretreated* $(n=24)$ & Docetaxel only $(n=95)$ & $p$ value \\
\hline Age, yr, mean $( \pm \mathrm{SD})$ & $64.8( \pm 19.3)$ & $67.5( \pm 7.2)$ & 0.5 \\
\hline White race, no. (\%) & $20(83)$ & $64(67)$ & 0.2 \\
\hline \multicolumn{4}{|l|}{ Gleason score, no. (\%) } \\
\hline 6 & $1(5)$ & $6(7)$ & \\
\hline 7 & $6(27)$ & $23(26)$ & \\
\hline $8-10$ & $15(68)$ & $59(67)$ & 0.9 \\
\hline Prior antiandrogen therapy ${ }^{* *}$, no. $(\%)$ & $22(92)$ & $83(87)$ & 0.6 \\
\hline Prior ketoconazole therapy, no. (\%) & $6(25)$ & $35(37)$ & 0.3 \\
\hline Docetaxel given weekly (as opposed to every $3 \mathrm{wk})^{\#}$, no. (\%) & $0(0)$ & $4(4)$ & 0.6 \\
\hline Antiosteoclast agent, no. (\%) & $17(71)$ & $45(47)$ & 0.07 \\
\hline \multicolumn{4}{|l|}{ ECOG performance status ${ }^{\dagger}$, no. $(\%)$} \\
\hline 0 & $5(33)$ & $42(53)$ & \\
\hline 1 & $10(67)$ & $27(34)$ & \\
\hline 2 & $0(0)$ & $10(13)$ & 0.04 \\
\hline Pain score, mean $( \pm \mathrm{SD})$ & $2.8( \pm 2.7)$ & $1.7( \pm 2.4)$ & 0.1 \\
\hline Hemoglobin level, g/dl, mean $( \pm$ SD) & $11.1( \pm 2.5)$ & $11.7( \pm 1.7)$ & 0.4 \\
\hline Albumin level, g/dl, mean $( \pm \mathrm{SD})$ & $3.9( \pm 0.4)$ & $4.3( \pm 1)$ & 0.003 \\
\hline Alkaline phosphatase level, mean $( \pm \mathrm{SD})$ & $265.5( \pm 290.8)$ & $277.7( \pm 402)$ & 0.9 \\
\hline Platelet count, thousands/ $/ \mu$, mean $( \pm \mathrm{SD})$ & $257.9( \pm 117.6)$ & $256.9( \pm 96.7)$ & 1 \\
\hline WBC count, $\mu \mathrm{l}$, mean $( \pm \mathrm{SD})$ & $7429.5( \pm 2619.6)$ & $8281.3( \pm 3533)$ & 0.2 \\
\hline Serum creatinine level, mg/dl, mean $( \pm \mathrm{SD})$ & $1( \pm 0.2)$ & $1( \pm 0.4)$ & 0.6 \\
\hline AST level, IU/l, mean ( \pm SD) & $36.6( \pm 39.2)$ & $33.2( \pm 27.7)$ & 0.7 \\
\hline ALT level, IU/l, mean $( \pm \mathrm{SD})$ & $30.1( \pm 31.6)$ & $23.3( \pm 15.2)$ & 0.4 \\
\hline \multicolumn{4}{|l|}{ Bone metastases, no (\%) } \\
\hline 0 & $1(5)$ & $4(4)$ & \\
\hline $1-3$ & $7(33)$ & $88(95)$ & \\
\hline $4-10$ & $2(10)$ & $1(1)$ & \\
\hline$>10$ & $11(52)$ & $0(0)$ & 1 \\
\hline Lymph node metastases, no. (\%) & $15(71)$ & $61(66)$ & 0.8 \\
\hline Lung metastases, no. (\%) & $8(40)$ & $16(17)$ & 0.04 \\
\hline Liver metastases, no. (\%) & $4(20)$ & $10(11)$ & 0.3 \\
\hline Baseline PSA level & $269.2( \pm 245.9)$ & $462.7( \pm 815.7)$ & 0.05 \\
\hline
\end{tabular}

$\mathrm{ALT}=$ alanine transaminase; $\mathrm{AST}=$ aspartate transaminase $; \mathrm{ECOG}=$ Eastern Cooperative Oncology Group; $\mathrm{PSA}=$ prostate-specific antigen; $\mathrm{SD}=$ standard deviation; $\mathrm{WBC}=$ white blood cell; .

* Patients treated with abiraterone before docetaxel.

*** Antiandrogen therapy refers to a first-generation androgen-receptor antagonist (eg, flutamide, bicalutamide, nilutamide).

${ }^{\#}$ With the exception of four patients in the docetaxel-alone cohort (who received weekly docetaxel), all patients received docetaxel $75 \mathrm{mg} / \mathrm{m}^{2}$ every $3 \mathrm{wk}$. 
${ }^{\dagger}$ There were no patients with ECOG performance status 3 or 4 . 


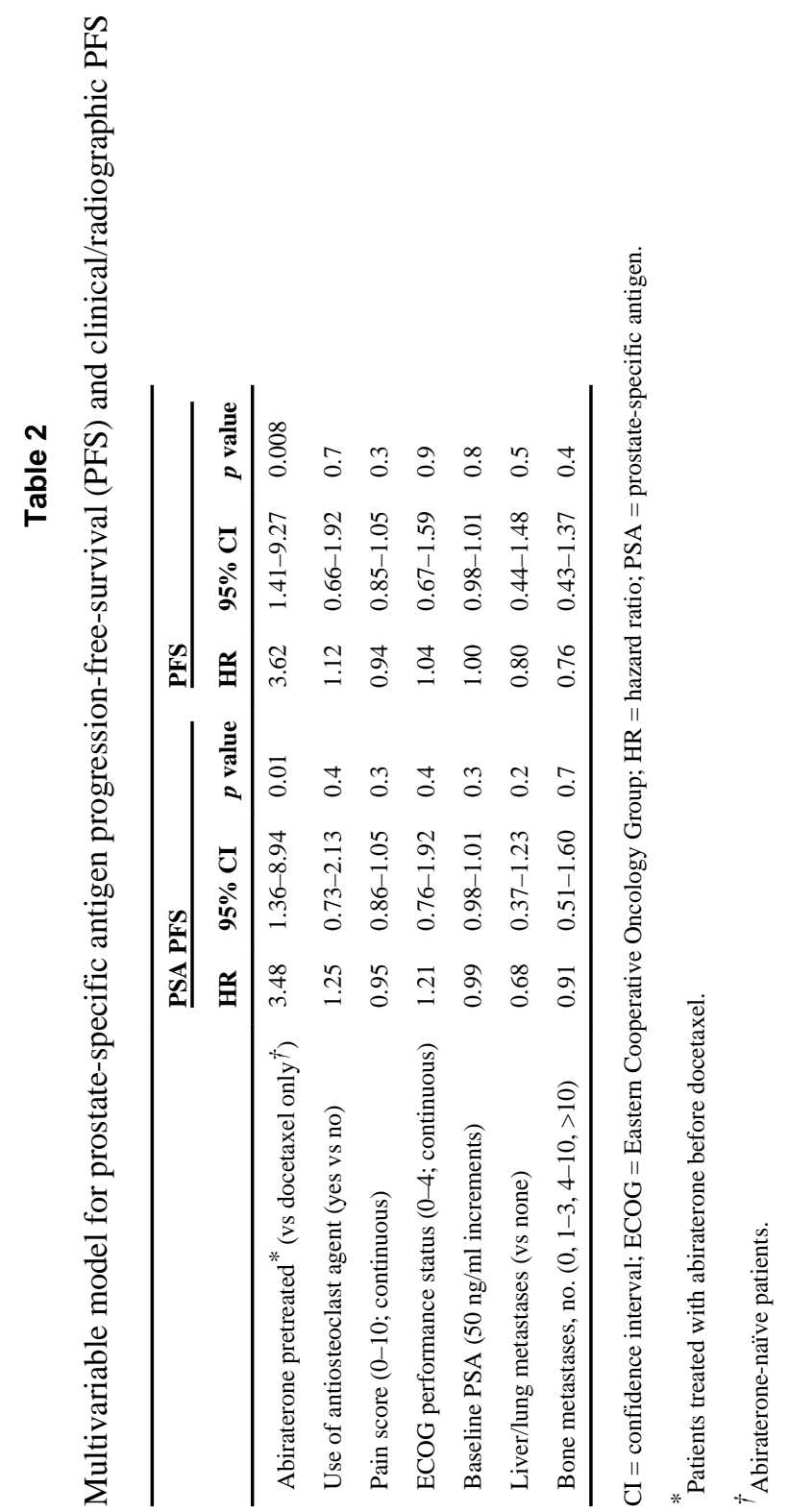

Eur Urol. Author manuscript; available in PMC 2015 October 01. 